\title{
Capturing Deuteration Effects in a Molecular Mechanics Force Field: Deuterated THF and the THF-Water Miscibility Gap
}

Rupesh Agarwal ${ }^{1,2}$, Micholas Dean Smith ${ }^{1,3^{*}}$, Jeremy C. Smith ${ }^{1,3^{*}}$

${ }^{1}$ UT/ORNL Center for Molecular Biophysics, Oak Ridge National Laboratory, Tennessee 37831-6309; ${ }^{2}$ Graduate School of Genome Science and Technology, University of Tennessee, Knoxville, Tennessee 37996; ${ }^{3}$ Department of Biochemistry and Cellular and Molecular Biology, University of Tennessee, Knoxville, Tennessee 37996

*co-corresponding authors

\section{Supplementary Information}

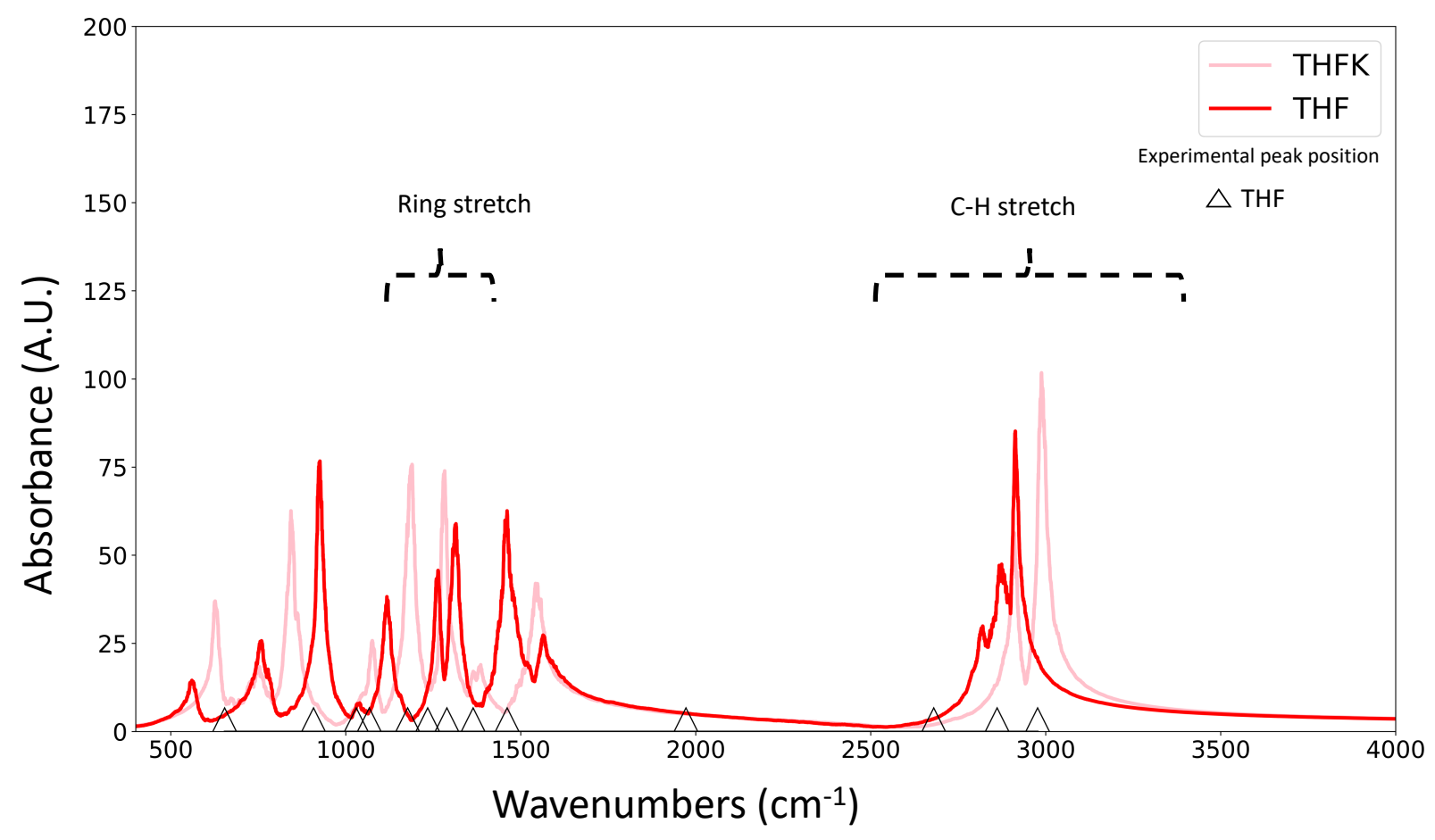

Fig S1: Infrared spectra of THF and THFK as calculated by MD simulations (lines) and experiments (peaks) 

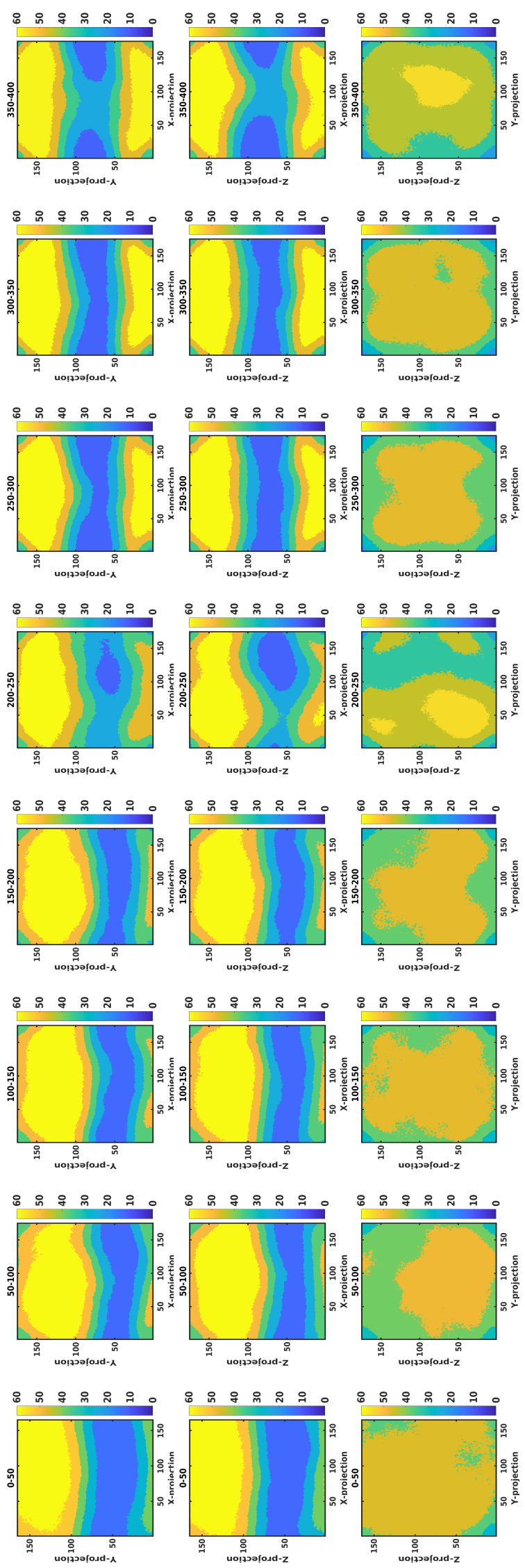

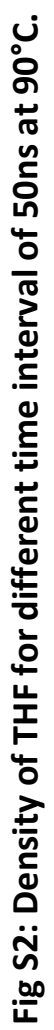



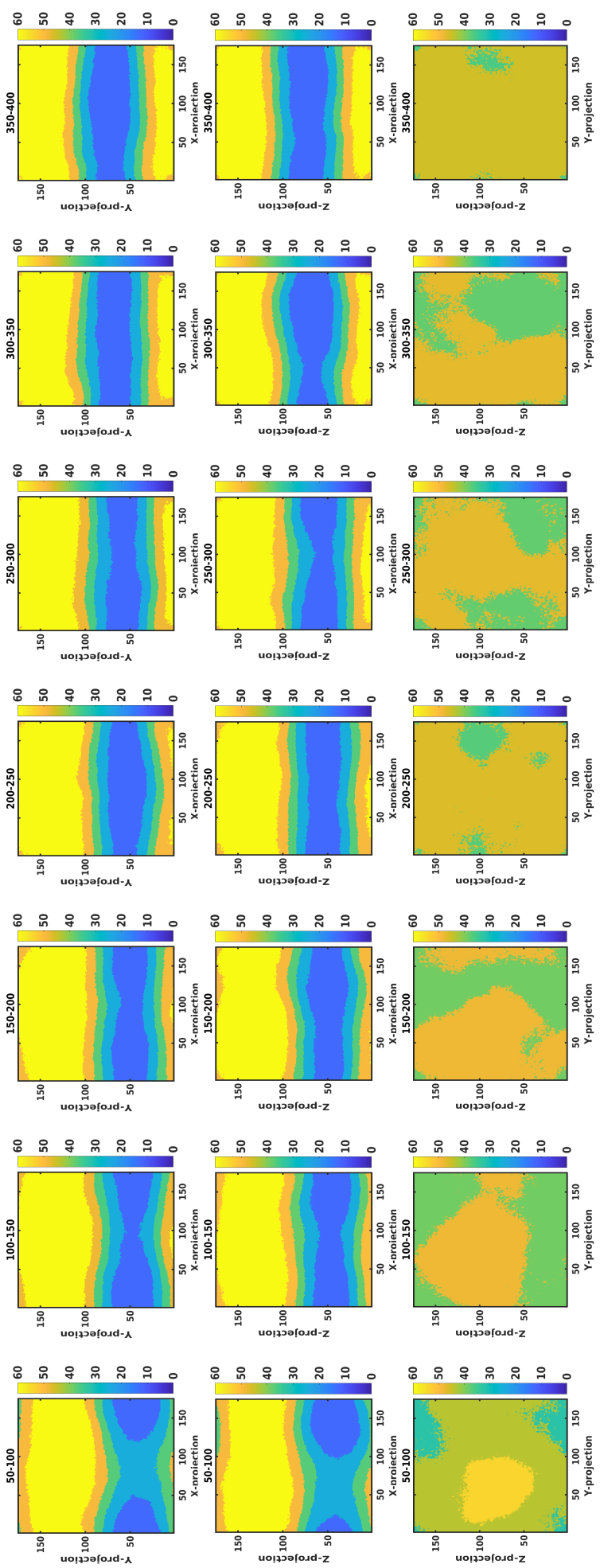

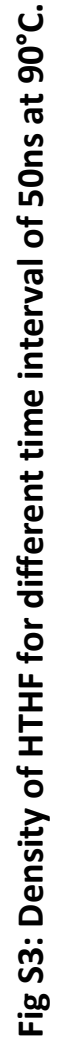

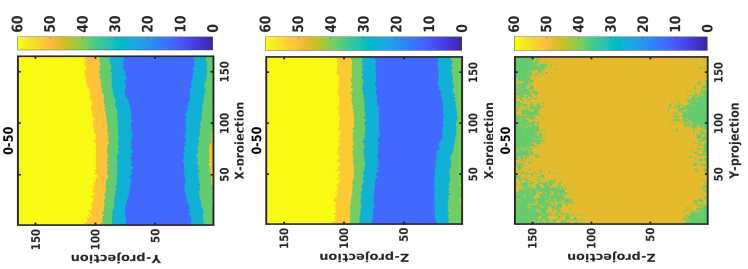



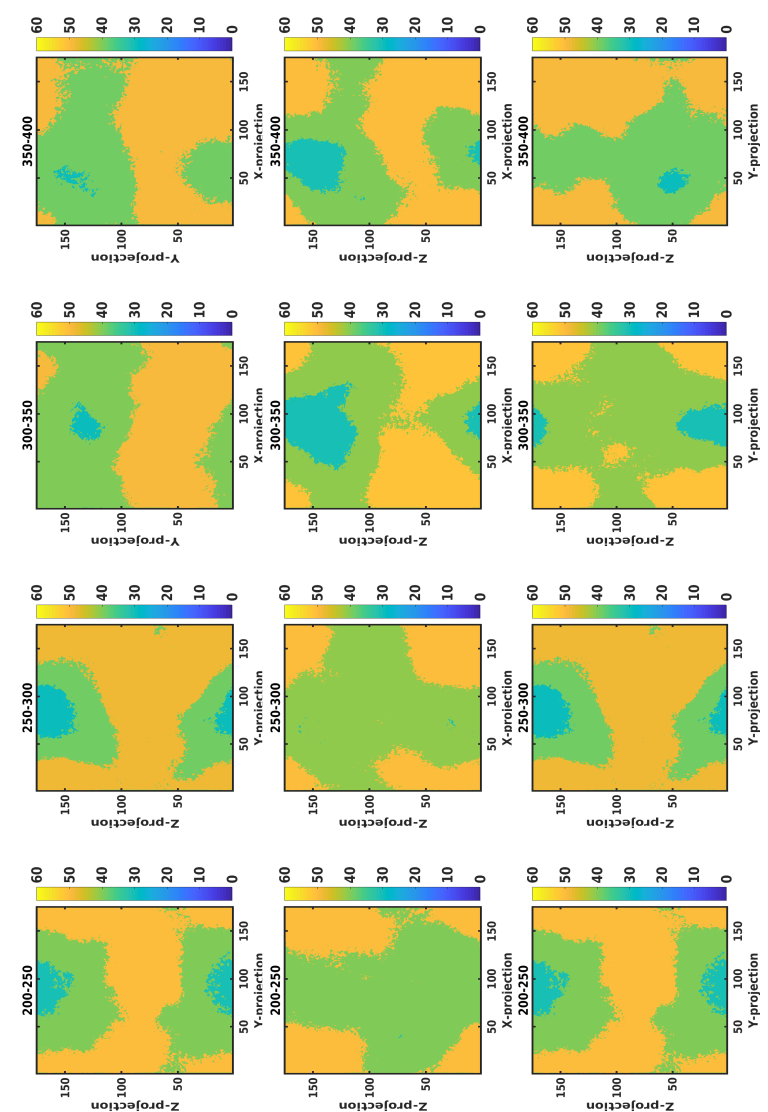

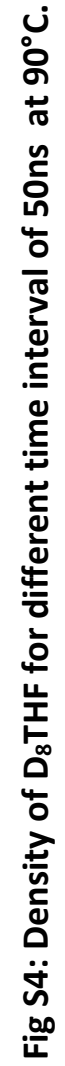

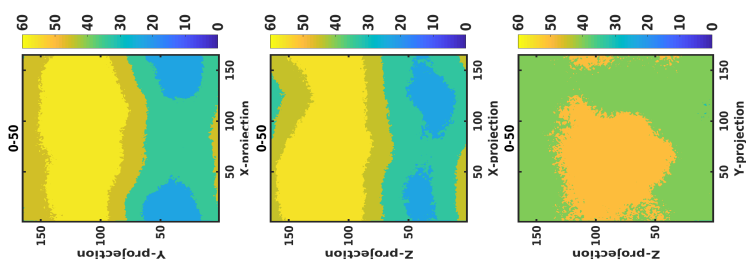



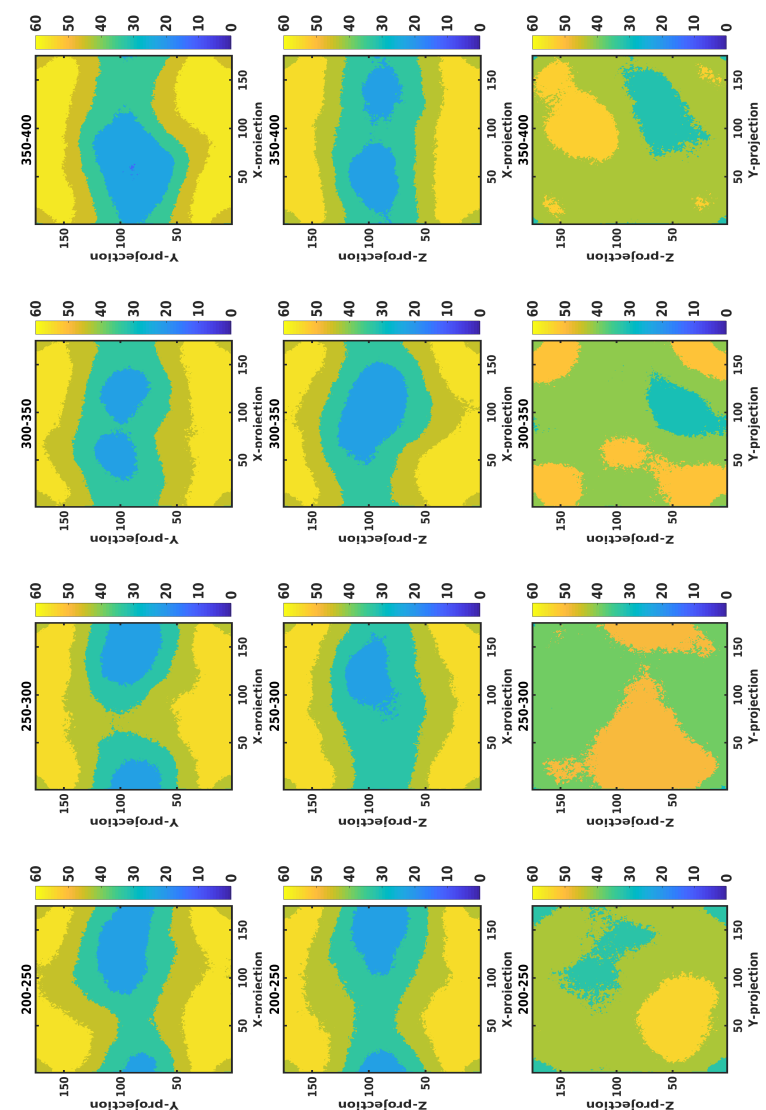

ن
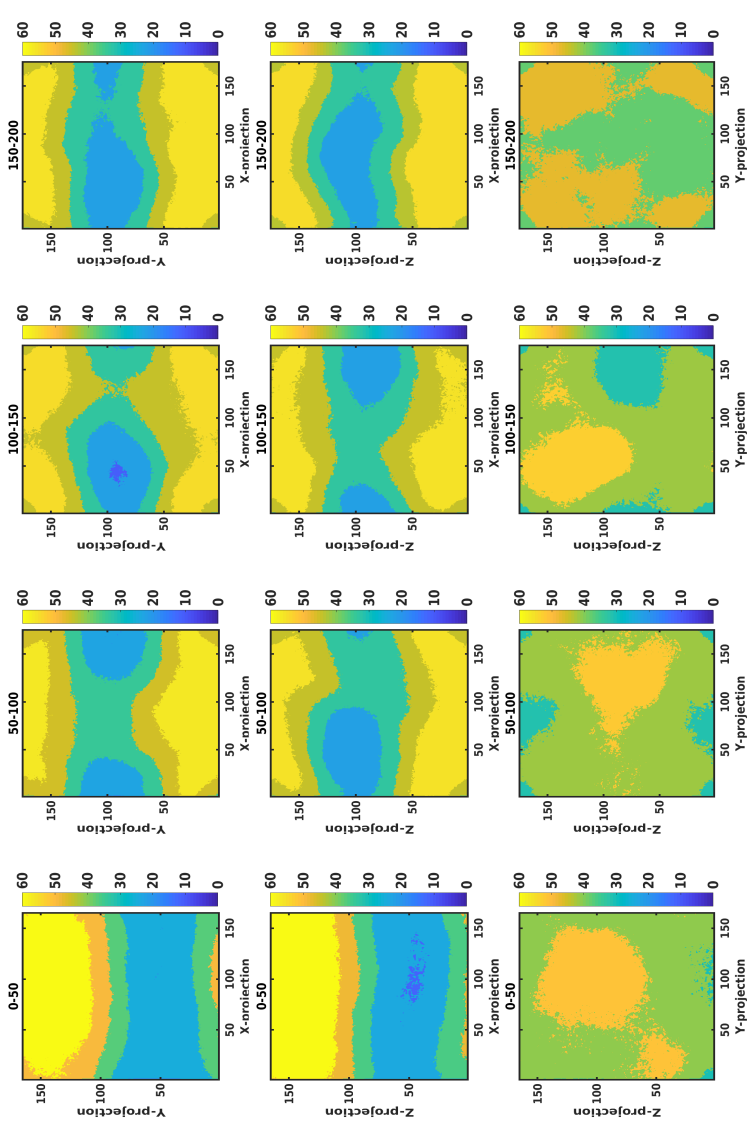

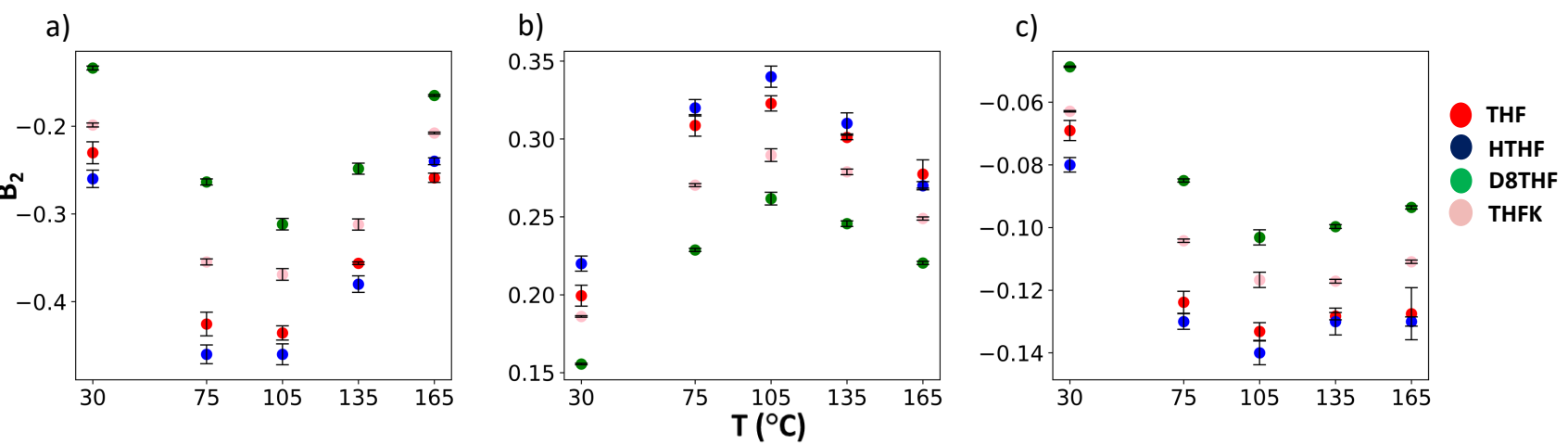

Figure S6: Individual components of the second virial coefficient: a) $D_{8}$ THF-D 8 THF, HTHF-HTHF, THF- THF, and THFK-THFK b) D8THF, HTHF, THFK and THF -water c) water-water. Error bars show the SEM, and those not visible are at most the size of the symbol.

a)

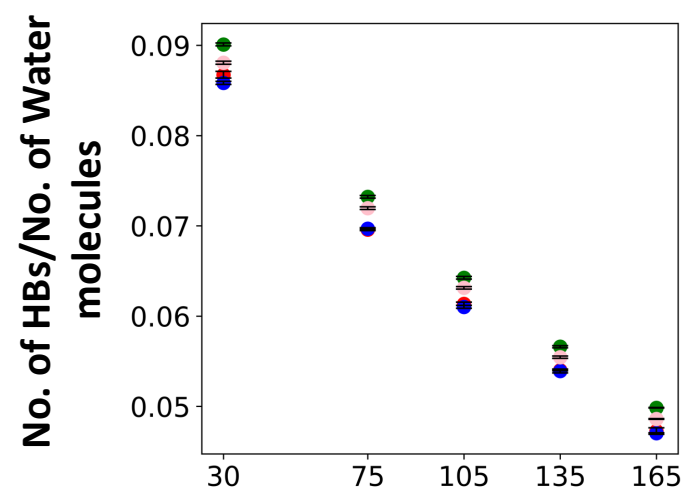

b)

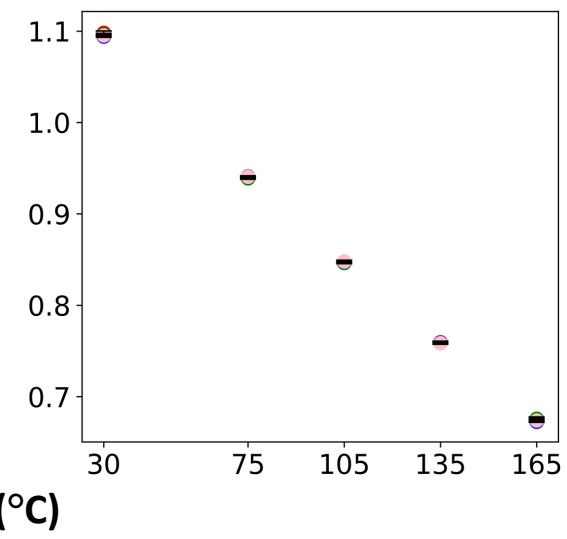

THF

HTHF

D8THF

THFK

Figure S7: Average number of hydrogen bonds normalized by the number of water molecules: a) D8THF, HTHF, THFK, and THF -water; b) water-water. Error bars show the SEM, and those not immediately visible are at most the size of the symbol. 
a)

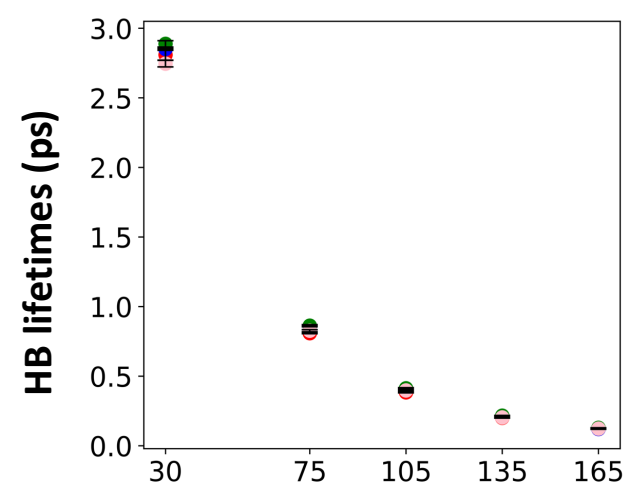

b)

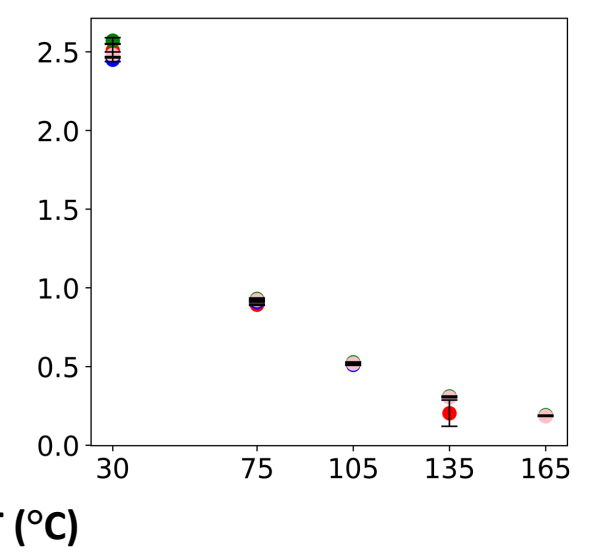

THF

HTHF

D8THF

THFK

Figure S8: Mean hydrogen bond lifetimes: a) $\mathrm{D}_{8}$ THF, HTHF, THFK, and THF -water; b) water-water. Error bars show the SEM, and those not immediately visible are at most the size of the symbol.
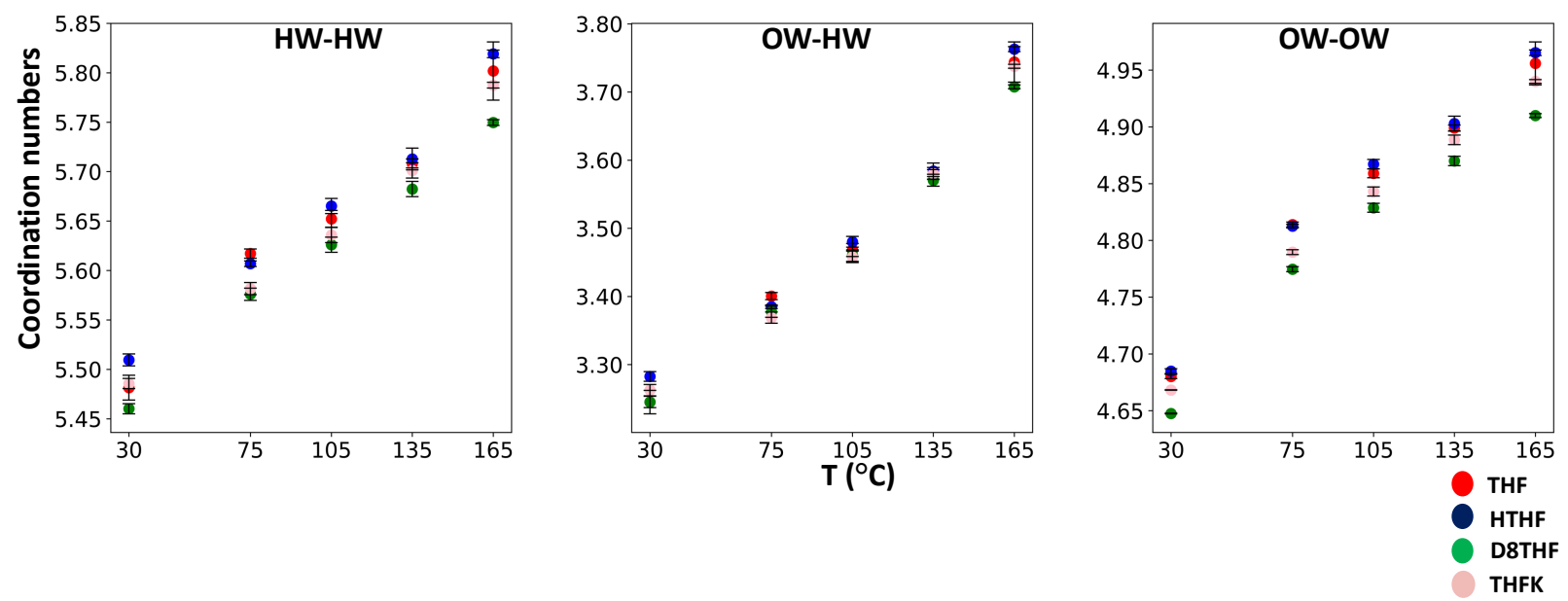

Figure S9: Co-ordination numbers between water-water. Error bars show the SEM, and those not immediately visible are at most the size of the symbol. 

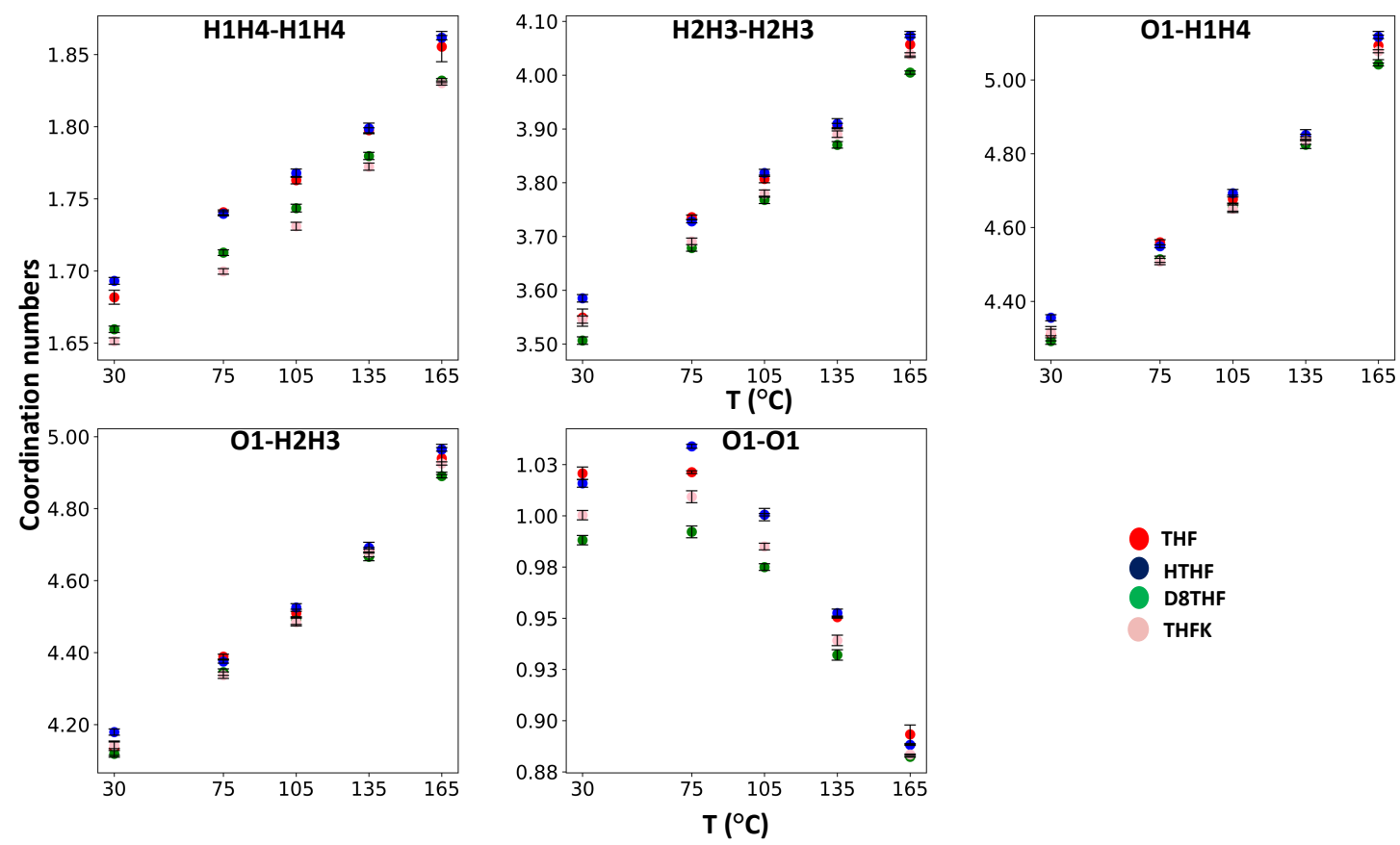

Figure S10: Co-ordination numbers between $\mathrm{D}_{8}$ THF- $\mathrm{D}_{8} \mathrm{THF}$, HTHF- HTHF, THFKTHFK, and THF-THF. Error bars show the SEM, and those not immediately visible are at most the size of the symbol.
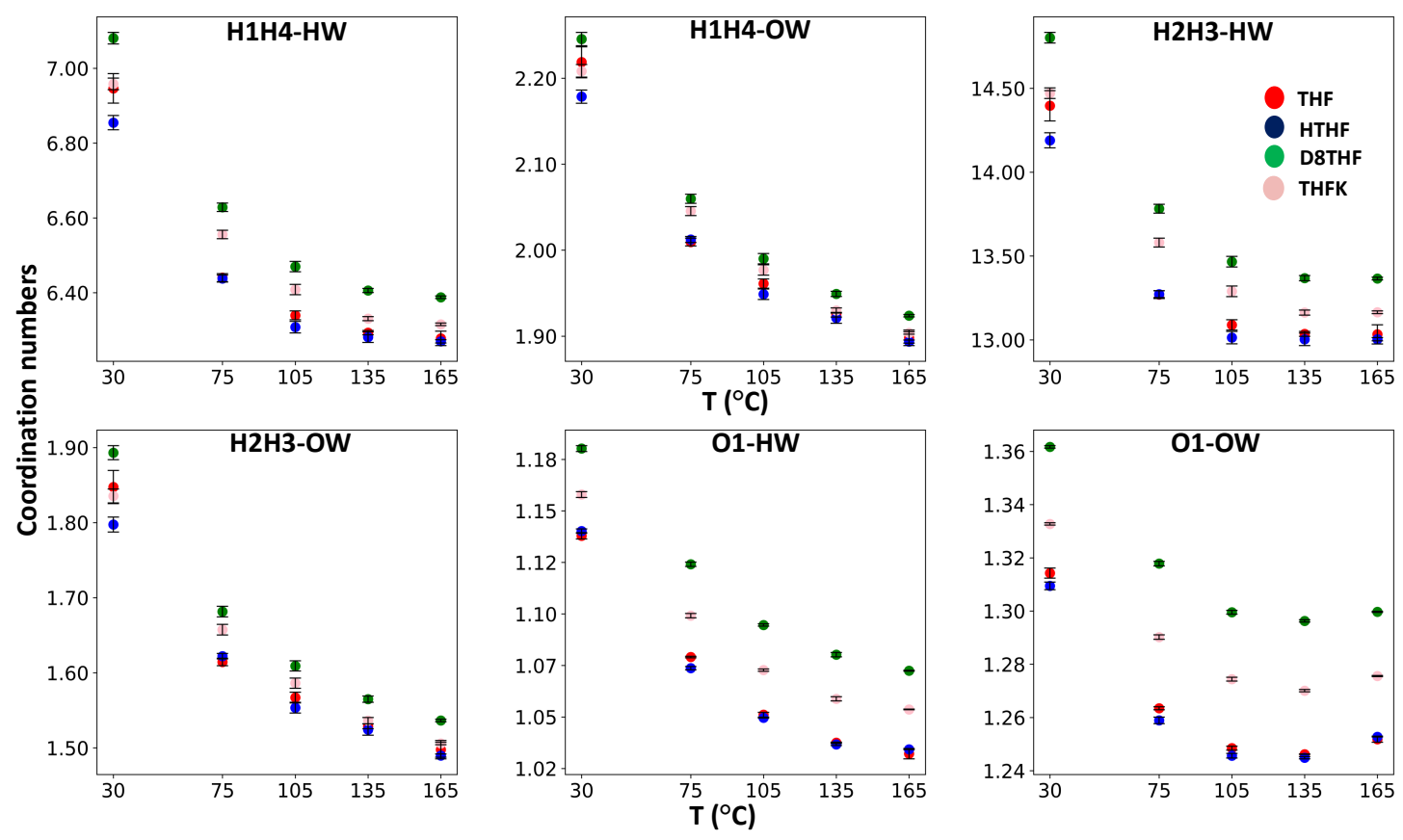
Figure S11: Co-ordination numbers between D $_{8}$ THF, HTHF, THFK, and THF -water. Error bars show the SEM, and those not immediately visible are at most the size of the symbol. 

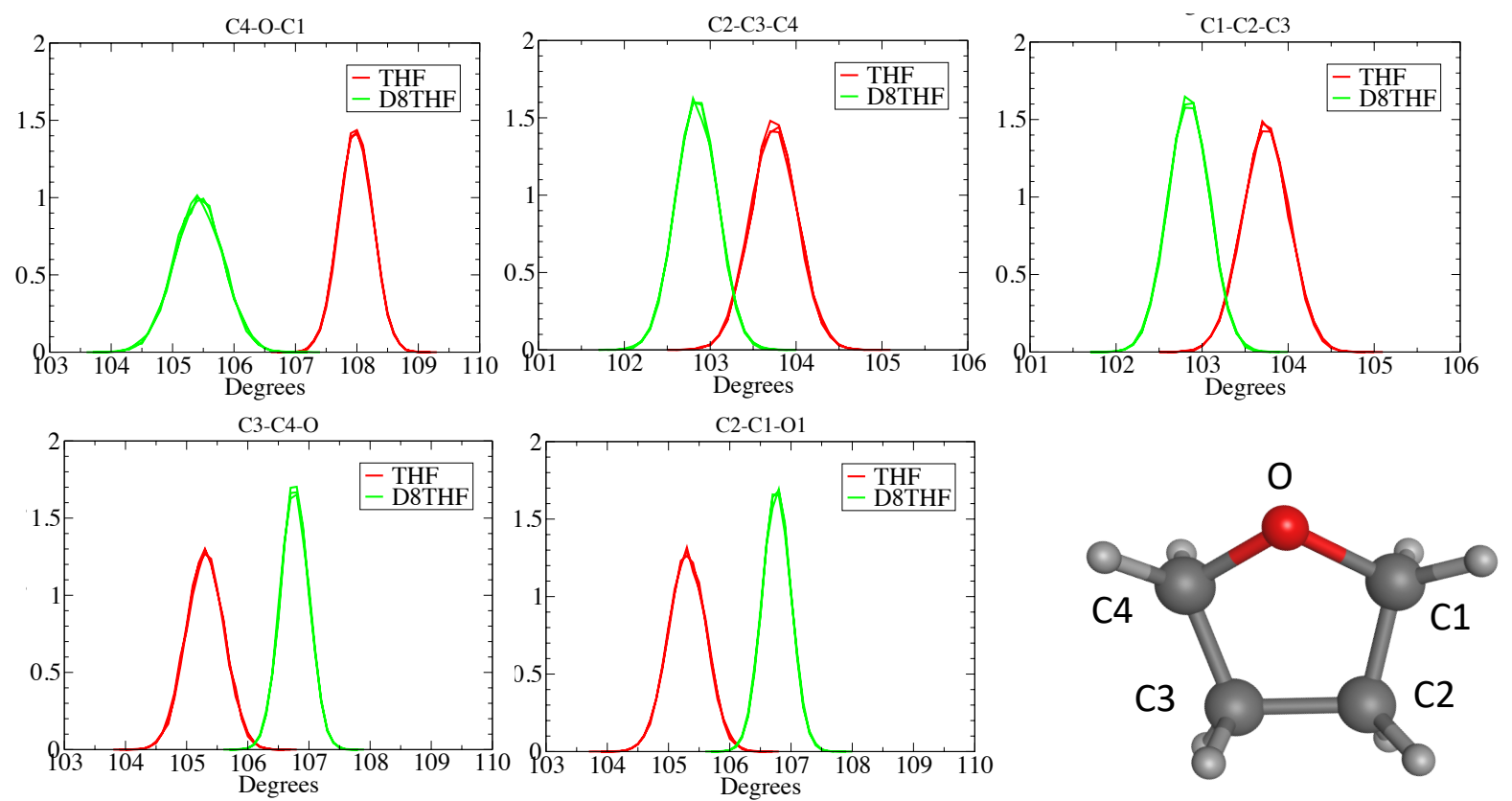

Fig S12: Probability distribution of angles for THF (red) and D8THF(green) in presence of water molecules at $105{ }^{\circ} \mathrm{C}$
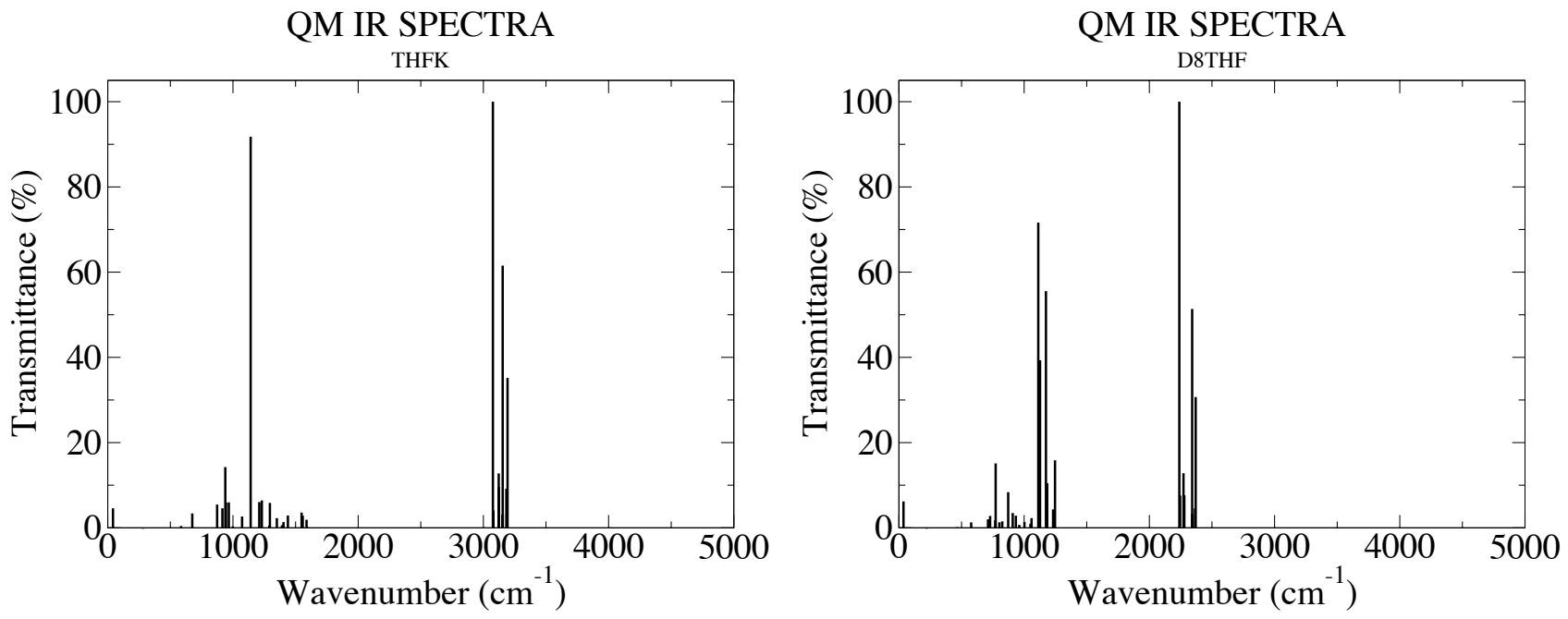

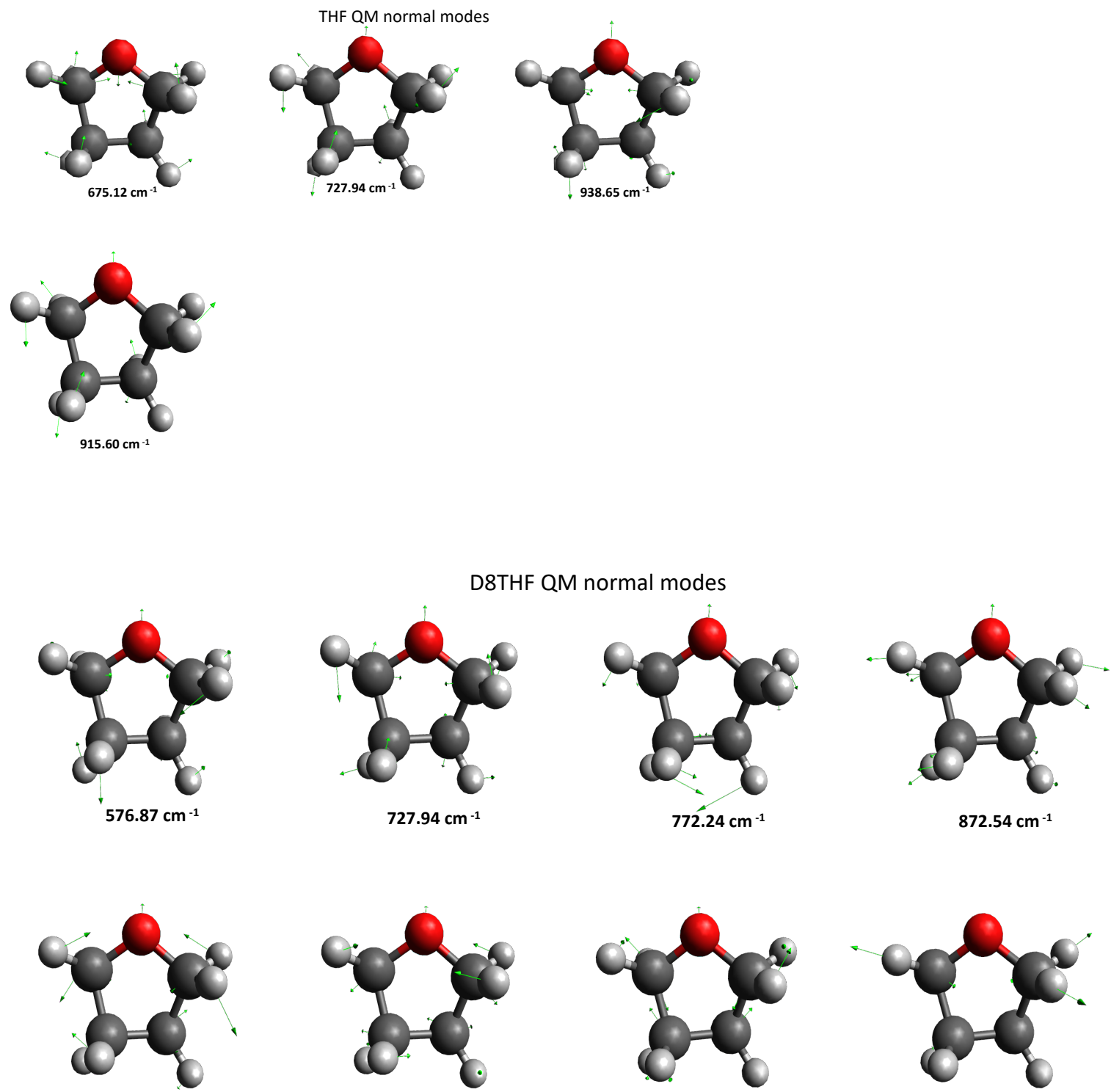

$933.54 \mathrm{~cm}^{-1}$

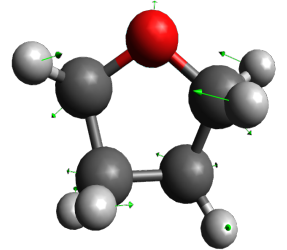

$1183.98 \mathrm{~cm}^{-1}$

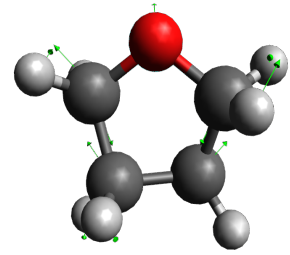

$1246.64 \mathrm{~cm}^{-1}$

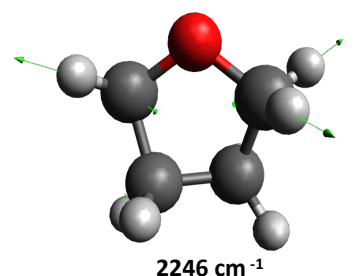

Fig S13: QM IR spectra (top) and corresponding QM normal modes for THF and D8THF (bottom) 
SI table 1: Bonded terms of new $D_{8}$ THF parameters

\begin{tabular}{|c|c|c|c|c|}
\hline Atom type & Atom type & $\mathbf{K}_{\mathbf{b}}$ & $\mathbf{b}_{\mathbf{0}}$ & $\mathrm{V}_{(\text {bond })}=\mathrm{K}_{\mathrm{b}}\left(\mathrm{b}-\mathrm{b}_{0}\right)^{2}$ \\
\hline $\mathrm{OC} 305 \mathrm{~A}$ & $\mathrm{CC} 325 \mathrm{~B}$ & 267.635 & 1.441 & $\mathrm{~K}_{\mathrm{b}}: \mathrm{kcal} / \mathrm{mole} / \AA^{2}$ \\
\hline $\mathrm{CC} 325 \mathrm{~B}$ & $\mathrm{CC} 325 \mathrm{~B}$ & 241.951 & 1.523 & $\mathrm{~b}_{0}: \AA$
\end{tabular}

SI table 2: Angle term of new $D_{8} T H F$ parameters

\begin{tabular}{|c|c|c|c|c|c|}
\hline Atom type & Atom type & Atom type & $\mathbf{K}_{\theta}$ & $\theta_{0}$ & \multirow{7}{*}{$\begin{array}{l}\mathrm{V}_{\text {(angle) }}=\mathrm{K}_{\theta}\left(\theta-\theta_{0}\right)^{2} \\
\mathrm{~K}_{\theta}: \mathrm{kcal} / \mathrm{mole} / \mathrm{rad}^{2} \\
\theta_{0}: \text { degrees }\end{array}$} \\
\hline OC305A & CC325B & $\mathrm{HCA} 25 \mathrm{~A}$ & 62.999 & 108.462 & \\
\hline OC305A & CC325B & CC325B & 141.069 & 106.72 & \\
\hline CC $325 B$ & CC325B & HCA25A & 44.901 & 110.984 & \\
\hline CC325B & CC325B & CC325B & 108.129 & 100.701 & \\
\hline CC $325 B$ & OC305A & CC $325 B$ & 32.544 & 110.792 & \\
\hline HCA25A & CC325B & HCA25A & 24.863 & 107.157 & \\
\hline
\end{tabular}

SI table 3: Dihedral term for new $D_{8}$ THF parameters

\begin{tabular}{|c|c|c|c|c|c|c|c|}
\hline $\begin{array}{l}\text { Atom } \\
\text { type }\end{array}$ & $\begin{array}{c}\text { Atom } \\
\text { type }\end{array}$ & $\begin{array}{c}\text { Atom } \\
\text { type }\end{array}$ & $\begin{array}{l}\text { Atom } \\
\text { type }\end{array}$ & $\mathbf{K} \chi$ & $\mathbf{n}$ & $\boldsymbol{\delta}$ & $\mathrm{V}_{(\text {dihedral) }}=\mathrm{K} \chi(1+\cos (\mathrm{n}(\chi)-\delta))$ \\
\hline HCA25A & CC325B & CC325B & HCA25A & 2.644 & 1 & 0 & Kx: kcal/mole \\
\hline CC325B & $\mathrm{CC} 325 \mathrm{~B}$ & $\mathrm{CC} 325 \mathrm{~B}$ & $\mathrm{CC} 325 \mathrm{~B}$ & 2.597 & 1 & 180 & n: multiplicity \\
\hline CC325B & OC305A & $\mathrm{CC} 325 \mathrm{~B}$ & CC325B & 1.148 & 1 & 180 & delta: degrees \\
\hline CC325B & $\mathrm{CC} 325 \mathrm{~B}$ & $\mathrm{CC} 325 \mathrm{~B}$ & OC305A & 2.992 & 1 & 180 & \\
\hline CC325B & CC325B & $\mathrm{CC} 325 \mathrm{~B}$ & HCA25A & 2.465 & 1 & 0 & \\
\hline HCA25A & $\mathrm{CC} 325 \mathrm{~B}$ & CC325B & OC305A & 2.807 & 1 & 180 & \\
\hline CC325B & OC305A & CC325B & HCA25A & 2.423 & 1 & 180 & \\
\hline
\end{tabular}

SI table 4: Lennard-Jones potential term of new $D_{8}$ THF parameters

\begin{tabular}{|l|c|c|c|c|c|}
\hline Atom type & epsilon & $\mathbf{R}_{\mathbf{m i n}} / \mathbf{2}$ & Ignored & $\mathbf{e p s}_{\mathbf{1 - 4}}$ & $\mathbf{R}_{\mathbf{m i n}} / \mathbf{2}_{\mathbf{1 - 4}}$ \\
\hline CC325B & -0.06 & 2.02 & 0 & -0.01 & 1.9 \\
\hline HCA25A & -0.035 & 1.3 & & & \\
\hline OC305A & -0.1 & 1.65 & & & \\
\hline
\end{tabular}


SI table 5: Bonded terms of new THFK parameters

\begin{tabular}{|c|c|c|c|}
\hline Atom type & Atom type & $\mathbf{K}_{\mathbf{b}}$ & $\mathbf{b}_{\mathbf{0}}$ \\
\hline OC305A & CC325B & 262.599 & 1.438 \\
\hline CC325B & CC325B & 241.762 & 1.526 \\
\hline CC325B & HCA25A & 345.662 & 1.095 \\
\hline
\end{tabular}

$$
\begin{aligned}
& \mathrm{V}_{\text {(bond) }}=\mathrm{K}_{\mathrm{b}}\left(\mathrm{b}-\mathrm{b}_{0}\right)^{2} \\
& \mathrm{~K}_{\mathrm{b}}: \mathrm{kcal} / \mathrm{mole}^{2} \AA^{2} \\
& \mathrm{~b}_{0}: \AA
\end{aligned}
$$

\begin{tabular}{|c|c|c|c|c|c|}
\hline Atom type & Atom type & Atom type & $\mathbf{K}_{\theta}$ & $\theta_{0}$ & \multirow{7}{*}{$\begin{array}{l}\mathrm{V}_{\text {(angle) }}=\mathrm{K}_{\theta}\left(\theta-\theta_{0}\right)^{2} \\
\mathrm{~K}_{\theta}: \mathrm{kcal} / \mathrm{mole} / \mathrm{rad}^{2} \\
\theta_{0}: \text { degrees }\end{array}$} \\
\hline OC $305 A$ & CC325B & HCA25A & 58.594 & 108.351 & \\
\hline OC305A & CC $325 B$ & CC $325 B$ & 146.195 & 106.406 & \\
\hline CC325B & CC325B & HCA25A & 36.183 & 110.953 & \\
\hline CC $325 B$ & CC $325 B$ & $\mathrm{CC} 325 \mathrm{~B}$ & 109.996 & 101.417 & \\
\hline CC $325 B$ & OC305A & CC $325 B$ & 41.030 & 108.634 & \\
\hline $\mathrm{HCA} 25 \mathrm{~A}$ & CC325B & $\mathrm{HCA} 25 \mathrm{~A}$ & 42.288 & 107.785 & \\
\hline
\end{tabular}

SI table 6: Angle term of new THFK parameters

SI table 7: Dihedral term for new THFK parameters

\begin{tabular}{|c|c|c|c|c|c|c|}
\hline $\begin{array}{c}\text { Atom } \\
\text { type }\end{array}$ & $\begin{array}{c}\text { Atom } \\
\text { type }\end{array}$ & $\begin{array}{c}\text { Atom } \\
\text { type }\end{array}$ & $\begin{array}{c}\text { Atom } \\
\text { type }\end{array}$ & $\mathbf{K} \boldsymbol{\chi}$ & $\mathbf{n}$ & $\boldsymbol{\delta}$ \\
\hline $\mathrm{HCA} 25 \mathrm{~A}$ & $\mathrm{CC} 325 \mathrm{~B}$ & $\mathrm{CC} 325 \mathrm{~B}$ & $\mathrm{HCA} 25 \mathrm{~A}$ & 2.999 & 1 & 0 \\
\hline $\mathrm{CC} 325 \mathrm{~B}$ & $\mathrm{CC} 325 \mathrm{~B}$ & $\mathrm{CC} 325 \mathrm{~B}$ & $\mathrm{CC} 325 \mathrm{~B}$ & 2.996 & 1 & 180 \\
\hline $\mathrm{CC} 325 \mathrm{~B}$ & $\mathrm{OC} 305 \mathrm{~A}$ & $\mathrm{CC} 325 \mathrm{~B}$ & $\mathrm{CC} 325 \mathrm{~B}$ & 1.773 & 1 & 180 \\
\hline $\mathrm{CC} 325 \mathrm{~B}$ & $\mathrm{CC} 325 \mathrm{~B}$ & $\mathrm{CC} 325 \mathrm{~B}$ & $\mathrm{OC} 305 \mathrm{~A}$ & 2.996 & 1 & 180 \\
\hline $\mathrm{CC} 325 \mathrm{~B}$ & $\mathrm{CC} 325 \mathrm{~B}$ & $\mathrm{CC} 325 \mathrm{~B}$ & $\mathrm{HCA} 25 \mathrm{~A}$ & 0.993 & 1 & 0 \\
\hline $\mathrm{HCA} 25 \mathrm{~A}$ & $\mathrm{CC} 325 \mathrm{~B}$ & $\mathrm{CC} 325 \mathrm{~B}$ & $\mathrm{OC} 305 \mathrm{~A}$ & 0.037 & 1 & 180 \\
\hline $\mathrm{CC} 325 \mathrm{~B}$ & $\mathrm{OC} 305 \mathrm{~A}$ & $\mathrm{CC} 325 \mathrm{~B}$ & $\mathrm{HCA} 25 \mathrm{~A}$ & 3.000 & 1 & 180 \\
\hline
\end{tabular}

$\mathrm{V}_{(\text {dihedral })}=\mathrm{K} \chi(1+\cos (\mathrm{n}(\chi)-\delta))$ $\mathrm{K}$ : $\mathrm{kcal} / \mathrm{mole}$ $\mathrm{n}$ : multiplicity delta: degrees

SI table 8: Lennard-Jones potential term of new THFK parameters

\begin{tabular}{|c|c|c|c|c|c|}
\hline Atom type & epsilon & $\mathbf{R}_{\mathbf{m i n}} / \mathbf{2}$ & Ignored & $\mathbf{e p s}_{\mathbf{1 - 4}}$ & $\mathbf{R}_{\mathbf{m i n}} / \mathbf{2}_{\mathbf{1 - 4}}$ \\
\hline CC325B & -0.06 & 2.02 & 0 & -0.01 & 1.9 \\
\hline HCA25A & -0.035 & 1.3 & & & \\
\hline
\end{tabular}




\begin{tabular}{|l|l|l|l|l|l|}
\hline OC305A & -0.1 & 1.65 & & & \\
\hline
\end{tabular}

SI table 9: Cutoffs for Coordination Number Calculations (Cutoff = location of first minima in corresponding site-site RDFs)

\begin{tabular}{|c|c|c|}
\hline Site 1 & Site 2 & $\begin{array}{l}\text { Cut off } \\
(\text { nm })\end{array}$ \\
\hline THF-O1 & $\begin{array}{c}\text { water- } \\
\text { OW }\end{array}$ & 0.34 \\
\hline THF-O1 & $\begin{array}{c}\text { water- } \\
\text { HW }\end{array}$ & 0.25 \\
\hline THF-O1 & THF-O1 & 0.53 \\
\hline THF-O1 & $\begin{array}{c}\text { THF- } \\
\text { H1H4 }\end{array}$ & 0.38 \\
\hline THF-O1 & $\begin{array}{c}\text { THF- } \\
\text { H2H3 }\end{array}$ & 0.36 \\
\hline THF- & water- & 0.37 \\
\hline H1H4 & OW & \\
\hline THF- & water- & 0.41 \\
H1H4 & HW & \\
\hline THF- & THF- & 0.31 \\
H1H4 & H1H4 & \\
\hline THF- & THF- & 0.32 \\
H1H4 & H2H3 & \\
\hline THF- & water- & 0.37 \\
H2H3 & OW & \\
\hline THF- & water- \\
H2H3 & HW & 0.51 \\
\hline THF- & THF- \\
H2H3 & 0.35 \\
\hline OWW & $\begin{array}{c}\text { water- } \\
\text { OW }\end{array}$ \\
\hline & 0.37 \\
\hline
\end{tabular}




\begin{tabular}{|c|c|c|}
\hline $\begin{array}{c}\text { water- } \\
\text { OW }\end{array}$ & $\begin{array}{c}\text { water- } \\
\text { HW }\end{array}$ & 0.25 \\
\hline $\begin{array}{c}\text { water- } \\
\text { HW }\end{array}$ & $\begin{array}{c}\text { water- } \\
\text { HW }\end{array}$ & 0.31 \\
\hline
\end{tabular}

